\title{
Effect of Price Concession Strategy and Preservation Technology on Inventory Model for Decaying Products with Partial Backlogging and Price-Stock-Dependent Demand
}

\author{
Lakshmi Narayan De ${ }^{1}$ and Piyali Mallick ${ }^{2}$ \\ ${ }^{1}$ Department of Mathematics, Haldia Government College, India. \\ ORCID: 0000-0003-0371-1847 \\ ${ }^{2}$ Department of Mathematics, Government General Degree College, Kharagpur-II, India. \\ ORCID: 0000-0003-4940-1270
}

\begin{abstract}
This paper develops an economic order quantity model for deteriorating products that assumes price and stock depending demand. Shortages are permitted with partial backlogging. The proposed model focused on two things. The first one is the consideration of the fact that the deterioration rate can be reduced by the use of preservation technology investment and the second one is using the assumption that the unit purchase cost has a hostile bond with the order size to maximize the total profit. The idea of salvage/recover cost is considered and merged in this model. The solution technique of proposed optimization model is exemplified by a couple of numerical illustrations. Concavity of the average profit function is shown by plotting graphs. Sensitivity investigation is done to study the effect of changing the value of all parameters in the projected maximization model.
\end{abstract}

Keywords: Inventory, Partial backlogging, Preservation investment, Price Discount, Stock \& Price dependent demand.

\section{INTRODUCTION}

Inventory or stock of products/items is most challengeable to provide smooth administration in business enterprises and organization. So, concentration in the study of inventory management or control is constantly growing. Over the last few years, different inventory models have been explored by several researchers. Ford Harris [1] was the creator who first developed a classical economic order quantity (EOQ) model with constant demand.

One of the vital things in market is customer's demand, which directly depends upon different factors like display of the items/ level of inventory, retailing price of the product, time, advertisement, price rises etc. Till now, many inventory models have been introduced by several researchers assuming various types of demand rate together with other different aspects. Customers prefer to purchase products from a shop which has large piles of goods in its shelf space due to visibility and variety of items. In contrast, less stock of items might raise the perception that they are not so fresh. In many years, researchers \& practitioners have realised the fact that the demand for some items may be based on the stock on display. Levin et al. [2] pointed out that bulky piles of goods exhibited in a supermarket will lead the buyer to buy more. Stock dependent demand is usually suitable for different kinds of manufacturing sectors such as treated and raw food industry, garments (fabrics, costumes etc.,) industry, automobile industry and electronics \& electrical industry etc. Padmanabhan and Vrat [3] solved an inventory model for deteriorating items by a non-linear goal programming technique where demand is depending upon on stock of products on display. Giri et al. [4] created an EPQ model for deteriorating items having inventory level dependent consumption rate.

On the other hand, in several sectors particularly food sectors, the demand of raw food items such as vegetables, fruits, fishes, eggs, meat, dairy product, rice, wheat etc., in a shop and treated food in hotel or restaurant are price sensitive. Customers like to purchase from a shop which has low selling price. If the seller increases the retailing price of the product, the customers would move other shopping places to satisfy their demand. There are numerous studies have been done on the effect of price variations. Kotler [5] incorporated marketing strategies into inventory decisions and discussed the connection between economic order quantity and pricing decision. Again, thinking the importance of stock and price both, Urban and Baker [6] well-thought-out an EOQ model for multivariate price, time and stock-induced demand.

Deterioration of product is a key issue in the inventory control policy. We cannot disregard this in the current study. There are some items like milk, ice-cream, vegetables, dairy product, grocery items which deteriorates over time. Whitin [7] was the innovator, who first studied an EOQ model of deteriorating items. An inventory model of deteriorating items which deteriorates exponentially is developed by Ghare and Schrader [8].

So, inventory system of deteriorating items has been studied for a long time. but little is known about the effect of investing in reducing the rate deterioration. Hsu et al. [9] developed an inventory model where the retailer invests on the preservation technology. Lee and Dye [10] first formulated a deteriorating inventory model with stock dependent demand by allowing preservation technology cost as a decision variable. Mishra [11] developed an inventory model of instantaneous deteriorating items with controllable deterioration rate using preservation 
technology for a time dependent demand. Mushud et al. [12] developed an inventory model under the joint effect of trade credit and preservation technology. So, in this paper preservation technology is used to lessen deterioration rate by which seller can increase his income, please customer's demand and increase commercial affordability.

As in most of the research work, demand depends upon selling price and stock, so the order size of the customer will be affected directly. So, retailer can offer discount and other promotional offer to attract large number of customers, and hence demand. Less selling price creates high consumption rate and large no. of orders will be placed to customers and in this situation, all-unit rebate offer will be provided to the customer according to the size of the order. Goh and Sharafali [13] developed a pricing model with discount offer at random time. Taleizadeh and Pentico [14] introduced price discount concept in their model. In this field, Taleizadeh et al. [15], Shah \& Naik [16], Noori-darayan et al. [17], Pervin \& Roy [18] and Guiping et al. [19] did their work taking price discount into reflection. Alfares and Ghaithan [20] developed an inventory model for no shortage case under price discount facility. Shaikh et al. [21] modified Alfares and Ghaithan's [20] model considering shortages.

Our work is developed under the following considerations: i) practice of preservation technology to decrease deterioration of items ii) demand is price and stock sensitive iii) price concession facility. Our purpose is to determine the maximum profit of this model. The next part of the paper is planned to organize as cited. The supposition and symbolizations of the model are presented in section 2 . Resulting the section 3 , we have settled a mathematical optimization problem of this model. In section 4 , we give numerical solution procedure an algorithm for the proposed model. In section 5, some numerical examples and graphical representation are carried out. The sensitivity analysis is documented in section 6 . In the last, we conclude and give some impending research scope in section 7.

Comparison among some previous research works and our proposed work

\begin{tabular}{|l|c|c|c|c|c|}
\hline Author(s) & $\begin{array}{l}\text { Price } \\
\text { dependent } \\
\text { demand }\end{array}$ & $\begin{array}{l}\text { Stock } \\
\text { dependent } \\
\text { demand }\end{array}$ & $\begin{array}{l}\text { Shortages with } \\
\text { backlogging }\end{array}$ & $\begin{array}{l}\text { Preservation } \\
\text { technique }\end{array}$ & $\begin{array}{l}\text { Price } \\
\text { discount }\end{array}$ \\
\hline Goh \& Sharafali [13] & $\sqrt{ }$ & $\times$ & $\times$ & $\times$ & $\sqrt{ }$ \\
\hline Hsu et al.[9] & $\times$ & $\sqrt{ }$ & $\times$ & $\sqrt{ }$ & $\times$ \\
\hline Lee \& Dye [10] & $\times$ & $\times$ & $\sqrt{ }$ & $\sqrt{ }$ & $\times$ \\
\hline Mishra [11] & $\times$ & $\times$ & $\sqrt{ }$ & $\times$ & $\sqrt{ }$ \\
\hline Taleizaadeh \&Pentico [14] & $\times$ & $\times$ & $\sqrt{ }$ & $\sqrt{ }$ \\
\hline Taleizaadeh et al. [15] & $\sqrt{ }$ & $\times$ & $\times$ & $\times$ & $\sqrt{ }$ \\
\hline Alfares \& Ghaithan [20] & $\sqrt{ }$ & $\sqrt{ }$ & $\times$ & $\times$ & $\sqrt{ }$ \\
\hline Shah \& Naik [16] & $\sqrt{ }$ & $\sqrt{ }$ & $\times$ & $\sqrt{ }$ & $\times$ \\
\hline Noori-darayan et al. [17] & $\sqrt{ }$ & $\sqrt{ }$ & $\times$ & $\sqrt{ }$ & $\times$ \\
\hline Pervin \& Roy [18] & $\sqrt{ }$ & $\times$ & $\sqrt{ }$ & $\sqrt{ }$ \\
\hline Guiping et al. [19] & $\sqrt{ }$ & $\times$ & $\times$ & $\sqrt{ }$ \\
\hline Shaikh et al. [21] & $\times$ & $\times$ & $\times$ & $\sqrt{ }$ \\
\hline Mashud et al. [12] & $\times$ & $\times$ & $\times$ & $\times$ \\
\hline This paper & $\times$ & $\times$ & $\times$ & $\times$ \\
\hline
\end{tabular}




\section{ASSUMPTIONS AND NOTATIONS:}

The following assumptions and notations are considered to formulate the proposed model.

\section{II.I Assumptions:}

i) Single item inventory model is considered.

ii) The replenishment rate is infinite and lead time is zero.

iii) The planning horizon of the inventory system is infinite.

iv) Shortages are permitted and shortages are partially backlogged with rate $1 / 1+\delta(T-t)$, where $\delta$ is backlogging parameter and $(T-t)$ is the customer's queuing time to receive the product.

v) The demand $D$ of this model is linearly selling price and inventory level $(I(t)$ )-dependent. Though, during deficiency period, it depends solely on the price of item. i.e.,

$$
D=\left\{\begin{array}{ll}
\alpha-\beta p+\gamma I(t), & \text { when } I(t)>0 \\
\alpha-\beta p, & \text { when } I(t) \leq 0
\end{array},\right.
$$

where $\alpha, \beta, \gamma>0$ and $\alpha-\beta p>0$.

vi) $\theta$ is the constant deterioration rate without using preservation technology $(0 \leq \theta \leq 1)$.

vii) To decrease the deterioration consequence, preservation technology is used. The resulting rate of deterioration with the investment of preservation technology is $\theta \omega(\xi)$, where $\omega(\xi)$ is a decreasing function with $\omega^{\prime \prime}(\xi)>0$. Here we have considered $\omega(\xi)=$ $e^{-v \xi}, v>0$ and $\omega(\xi)=\frac{1}{1+v \xi}, v>0$.

viii) Purchasing price per unit item is a dropping phase function based on the ordering number $(Q) . Q_{i}, i=$ $1,2,3 \ldots, n+1\left(Q_{1}<Q_{2}<Q_{3}<\cdots<Q_{n}<Q_{n+1}=\right.$ $\infty)$ are sizes that govern the $n$ price breaks with in the unit purchase cost $C_{i}, i=1,2,3, \ldots, n\left(C_{1}>C_{2}>\cdots>\right.$ $\left.C_{n}\right)$.

\section{II.II Notations:}

i) $\quad K:$ replenishment cost per cycle

ii) $\quad \alpha:$ constant portion of the demand rate $(\alpha>0)$ iii) $\quad \beta$ : price dependent demand rate parameter $(\beta>0)$

iv) $\quad \gamma$ : stock-induced demand rate parameter $(\gamma>0)$

v) $\delta$ : backlogging parameter

vi) $\quad \theta$ : deterioration rate without preservation technology investment $(0 \leq \theta \leq 1)$

vii) $\quad p$ : retailing price per unit item

viii) $C_{h}$ : holding charge for per unit item per unit time

ix) $\quad C_{d}$ : deterioration cost per unit item

x) $\quad C_{i}:$ purchase cost per unit item

xi) $\quad C_{s}:$ Shortage cost per unit item

xii) $\quad C_{l}:$ lost sale cost per unit item

xiii) $S$ : initial stock of inventories in each cycle

xiv) $R$ : maximum deficiencies per cycle

xv) $Q:$ number of ordering quantity per replenishment cycle

xvi) $\quad \eta$ : salvage/ recover coefficient $(0 \leq \eta \leq 1)$

xvii) $\quad I_{1}(t)$ : Inventory level at time $t$ during positive stock period

xviii) $\quad I_{2}(t)$ : deficiency level at time $t$ during shortage period

xix) $A P$ : average profit per unit time

\section{Decision Variables}

i) $\quad T$ : Replenishment time per cycle

ii) $t_{1}$ : time at which inventory reaches zero

iii) $\xi$ : preservation charge per unit time

\section{MATHEMATICAL MODEL CONSTRUCTION}

The objective of the model is to determine the optimum turnover for items having above cited demand and deterioration. The replenishment of inventory is carried out at the beginning and eventually at the end of cycle. The level of inventory diminishes, during positive stock period occurs due to demand as well as the resulting deterioration rate and in the shortage period due to demand only, which is depicted in the Fig 1. The governing differential equation is as follows:

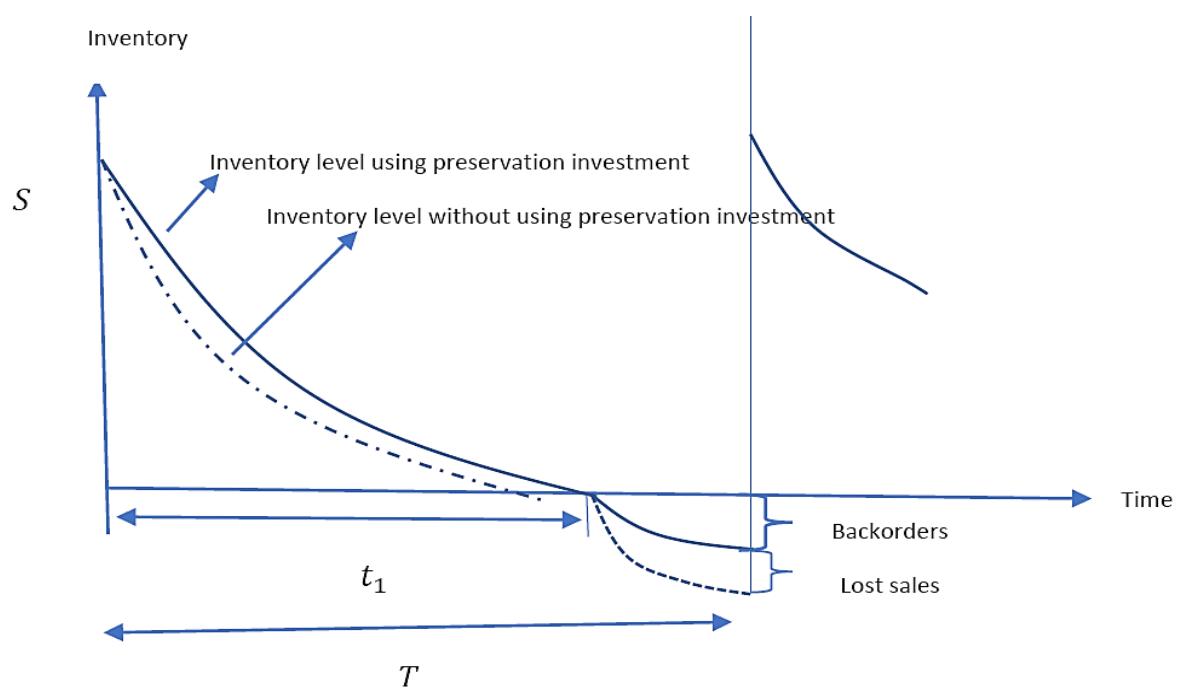

Fig1. Pictographic illustration of the model 


$$
\begin{aligned}
& \frac{d I_{1}(t)}{d t}+\theta \omega(\xi) I_{1}(t)=-\left(\alpha-\beta p+\gamma I_{1}(t)\right), \quad 0 \leq t \leq t_{1} \\
& \& \\
& \frac{d I_{2}(t)}{d t}=-\frac{(\alpha-\beta p)}{1+\delta(T-t)}, \quad t_{1}<t \leq T
\end{aligned}
$$

With boundary conditions $I_{1}(0)=S, I_{1}\left(t_{1}\right)=0$, and $I_{2}(T)=-R$.

The solution of equation (1) is

$$
I_{1}(t)=\frac{\alpha-\beta p}{\gamma+\theta \omega}\left(e^{(\gamma+\theta \omega)\left(t_{1}-t\right)}-1\right)
$$

and the solution of equation (2) is

$$
I_{2}(t)=\frac{\alpha-\beta p}{\delta}[\log (1+\delta(T-t))]-R
$$

Using the initial condition $I_{1}(0)=S$, from equation (3), we get

$$
S=\frac{\alpha-\beta p}{\gamma+\theta \omega}\left(e^{(\gamma+\theta \omega) t_{1}}-1\right)
$$

Using the initial condition $I_{1}\left(t_{1}\right)=0$, from equation (4), we get

$$
R=\frac{\alpha-\beta p}{\delta}\left[\log \left(1+\delta\left(T-t_{1}\right)\right)\right]
$$

So, total ordering quantity $Q=R+S=\frac{\alpha-\beta p}{\gamma+\theta \omega}\left(e^{(\gamma+\theta \omega) t_{1}}-1\right)+\frac{\alpha-\beta p}{\delta}\left[\log \left(1+\delta\left(T-t_{1}\right)\right)\right]$

Hence, the cycle length $T$ can be expressed as follows:

$$
\left.T=t_{1}+\frac{1}{\delta}\left[e^{\frac{\delta}{(\alpha-\beta p)}\left\{Q-\frac{(\alpha-\beta p)}{(\gamma+\theta \omega(\xi))}\left\{e^{(\gamma+\theta \omega(\xi)) t_{1}}-1\right\}\right.}\right\}-1\right]
$$

Now the total sales revenue $(S R)$ per cycle $=p \int_{0}^{t_{1}}\left\{\alpha-\beta p+\gamma I_{1}(t)\right\} d t+p \int_{t_{1}}^{T} \frac{(\alpha-\beta p)}{1+\delta(T-t)} d t$

$$
=(\alpha-\beta p) p t_{1}+\gamma p \frac{\alpha-\beta p}{\gamma+\theta \omega}\left[\frac{\left(e^{\left.(\gamma+\theta \omega) t_{1}-1\right)}\right.}{\gamma+\theta \omega}-t_{1}\right]+\frac{p(\alpha-\beta p)}{\delta} \log \left[1+\delta\left(T-t_{1}\right)\right]
$$

Replenishment cost $(R C)$ per cycle $=K$

Total inventory cost $(I H C)$ per cycle $=C_{h} \int_{0}^{t_{1}} I_{1}(t) d t=C_{h} \frac{\alpha-\beta p}{\gamma+\theta \omega}\left[\frac{\left(e^{(\gamma+\theta \omega) t_{1}}-1\right)}{\gamma+\theta \omega}-t_{1}\right]$

Total purchase cost $(P C)$ per cycle $=C_{i} Q=C_{i}\left\{\frac{\alpha-\beta p}{\gamma+\theta \omega}\left(e^{(\gamma+\theta \omega) t_{1}}-1\right)+\frac{\alpha-\beta p}{\delta}\left[\log \left(1+\delta\left(T-t_{1}\right)\right)\right]\right\}$

Total deterioration cost $(D C)$ per cycle $=C_{d} \cdot \theta \omega \int_{0}^{t_{1}} I_{1}(t) d t=C_{d} \cdot \theta \omega(\xi)\left\{\frac{\alpha-\beta p}{\gamma+\theta \omega}\left[\frac{\left(e^{\left.(\gamma+\theta \omega) t_{1}-1\right)}\right.}{\gamma+\theta \omega}-t_{1}\right]\right\}$

Total salvage value for the deteriorating items $(S V)$ per cycle $=\eta \cdot D C=\eta C_{d} \cdot \theta \omega\left\{\frac{\alpha-\beta p}{\gamma+\theta \omega}\left[\frac{\left(e^{\left.(\gamma+\theta \omega) t_{1}-1\right)}\right.}{\gamma+\theta \omega}-t_{1}\right]\right\}$

Total shortage cost $(S C)$ per cycle $=C_{S} \int_{t_{1}}^{T} I_{2}(t) d t=\frac{C_{S}(\alpha-\beta p)}{\delta}\left[\left(T-t_{1}\right)-\frac{\log \left\{1+\delta\left(T-t_{1}\right)\right\}}{\delta}\right]$

Total lost sale $\operatorname{cost}(L S C)$ per cycle $=C_{l}(\alpha-\beta p)\left[\left(T-t_{1}\right)-\frac{\log \left\{1+\delta\left(T-t_{1}\right)\right\}}{\delta}\right]$

Total preservation cost $(P R C)$ per cycle $=\xi T$

Average profit $\left(A P\left(T, t_{1}, \xi\right)\right)$ per unit time $=\frac{1}{T}\{S R+S V-R C-I H C-P C-D C-S C-L S C-P R C\}$

$$
=\frac{1}{T}\left[\begin{array}{c}
(\alpha-\beta p) p t_{1}+\left\{\gamma p+\left(C_{d}-\eta C_{d}\right) \theta \omega-C_{h}\right\}\left(\frac{\alpha-\beta p}{\gamma+\theta \omega}\right)\left\{\frac{\left(e^{(\gamma+\theta \omega) t_{1}-1}\right)}{\gamma+\theta \omega}-t_{1}\right\}-C_{i}\left(\frac{\alpha-\beta p}{\gamma+\theta \omega}\right)\left(e^{(\gamma+\theta \omega) t_{1}}-1\right) \\
+\frac{(\alpha-\beta p)}{\delta}\left(p-C_{i}+\frac{C_{s}}{\delta}+C_{l}\right) \log \left\{1+\delta\left(T-t_{1}\right)\right\}-(\alpha-\beta p)\left(\frac{C_{s}}{\delta}+C_{l}\right)\left(T-t_{1}\right)-\xi T-K
\end{array}\right]
$$


As stock-dependent parameter $(\gamma)$ and deterioration $(\theta)$ is small, so we expand the exponential term by Taylor expansion formula and overlooking third and higher order terms we write:

$A P\left(T, t_{1}, \xi\right)=\frac{1}{T}\left[\begin{array}{c}(\alpha-\beta p)\left(p-C_{i}+\frac{C_{s}}{\delta}+C_{l}\right) t_{1}+\frac{(\alpha-\beta p)}{2}\left\{\gamma p+\left(C_{d}-\eta C_{d}\right) \theta \omega(\xi)-C_{h}-C_{i}(\gamma+\theta \omega(\xi))\right\} t_{1}^{2} \\ +\frac{(\alpha-\beta p)}{\delta}\left(p-C_{i}+\frac{C_{s}}{\delta}+C_{l}\right) \log \left\{1+\delta\left(T-t_{1}\right)\right\}-\left\{(\alpha-\beta p)\left(\frac{C_{s}}{\delta}+C_{l}\right)+\xi\right\} T-K\end{array}\right]$

Now our objective is to obtain optimal cycle time $T^{*}$, preservation cost $\xi^{*}$ and $t_{1}^{*}$ in order to maximize the average profit $\left(A P\left(\xi, T, t_{1}\right)\right)$ per unit time.

In order to find out necessary conditions to maximize profit, we have

$\frac{\partial(A P)}{\partial T}=0$ i.e.,

$(\alpha-\beta p)\left(p-C_{i}+\frac{C_{s}}{\delta}+C_{l}\right) t_{1}+\frac{(\alpha-\beta p)}{2}\left\{\gamma p+\left(C_{d}-\eta C_{d}\right) \theta \omega(\xi)-C_{h}-C_{i}(\gamma+\theta \omega(\xi))\right\} t_{1}^{2}$

$$
+\frac{(\alpha-\beta p)}{\delta}\left(p-C_{i}+\frac{C_{s}}{\delta}+C_{l}\right) \log \left\{1+\delta\left(T-t_{1}\right)\right\}-\frac{\frac{(\alpha-\beta p)}{\delta}\left(p-C_{i}+\frac{C_{S}}{\delta}+C_{l}\right) T}{1+\delta\left(T-t_{1}\right)}=0
$$

$\frac{\partial(A P)}{\partial t_{1}}=0$ i.e.,

$(\alpha-\beta p)\left(p-C_{i}+\frac{C_{s}}{\delta}+C_{l}\right)+(\alpha-\beta p)\left\{\gamma p+\left(C_{d}-\eta C_{d}\right) \theta \omega(\xi)-C_{h}-C_{i}(\gamma+\theta \omega(\xi))\right\} t_{1}-\frac{\frac{(\alpha-\beta p)}{\delta}\left(p-C_{i}+\frac{C_{s}}{\delta}+C_{l}\right)}{1+\delta\left(T-t_{1}\right)}=0$

and

$\frac{\partial(A P)}{\partial \xi}=0$ i. e., $(\alpha-\beta p)\left\{\left(C_{d}-\eta C_{d}-C_{i}\right) \theta \omega^{\prime}(\xi)\right\} t_{1}^{2}-2 T=0$

Solving equations (19), (20) and (21) simultaneously, we can get $\xi^{*}, t_{1}^{*}$ and $T^{*}$.

The average total profit function $A P\left(T, t_{1}, \xi\right)$ achieves the global maximum value at $\left(T^{*}, t_{1}^{*}, \xi^{*}\right)$, if $A P\left(T, t_{1}, \xi\right)$ is strictly pseudoconcave (Cambini and Martein [22] and Dye [23]).

We write average profit function as $A P\left(T, t_{1}, \xi\right)=\frac{\varphi\left(T, t_{1}, \xi\right)}{\psi\left(T, t_{1}, \xi\right)}$, where

$\varphi\left(T, t_{1}, \xi\right)=\left[\begin{array}{c}(\alpha-\beta p)\left(p-C_{i}+\frac{C_{s}}{\delta}+C_{l}\right) t_{1}+\frac{(\alpha-\beta p)}{2}\left\{\gamma p+\left(C_{d}-\eta C_{d}\right) \theta \omega(\xi)-C_{h}-C_{i}(\gamma+\theta \omega(\xi))\right\} t_{1}^{2} \\ +\frac{(\alpha-\beta p)}{\delta}\left(p-C_{i}+\frac{C_{s}}{\delta}+C_{l}\right) \log \left\{1+\delta\left(T-t_{1}\right)\right\}-\left\{(\alpha-\beta p)\left(\frac{C_{s}}{\delta}+C_{l}\right)+\xi\right\} T-K\end{array}\right]$

and $\psi\left(T, t_{1}, \xi\right)=T$

$\frac{\partial^{2} \varphi}{\partial T^{2}}=-(\alpha-\beta p) \delta \frac{\left(p-C_{i}+\frac{C_{S}}{\delta}+C_{l}\right)}{\left\{1+\delta\left(T-t_{1}\right)\right\}^{2}}$

$\frac{\partial^{2} \varphi}{\partial t_{1}^{2}}=(\alpha-\beta p)\left\{\gamma p+\left(C_{d}-\eta C_{d}\right) \theta \omega(\xi)-C_{h}-C_{i}(\gamma+\theta \omega(\xi))\right\}-(\alpha-\beta p) \delta \frac{\left(p-C_{i}+\frac{C_{S}}{\delta}+C_{l}\right)}{\left\{1+\delta\left(T-t_{1}\right)\right\}^{2}}$

$\frac{\partial^{2} \varphi}{\partial \xi^{2}}=\frac{(\alpha-\beta p)}{2}\left\{\gamma p+\left(C_{d}-\eta C_{d}\right) \theta \omega^{\prime \prime}(\xi)-C_{h}-C_{i}\left(\gamma+\theta \omega^{\prime \prime}(\xi)\right)\right\} t_{1}^{2}$

$\frac{\partial^{2} \varphi}{\partial T \partial t_{1}}=\frac{\partial^{2} \varphi}{\partial t_{1} \partial T}=(\alpha-\beta p) \delta \frac{\left(p-C_{i}+\frac{C_{S}}{\delta}+C_{l}\right)}{\left\{1+\delta\left(T-t_{1}\right)\right\}^{2}}$

$\frac{\partial^{2} \varphi}{\partial T \partial \xi}=\frac{\partial^{2} \varphi}{\partial \xi \partial T}=-1$

$\frac{\partial^{2} \varphi}{\partial \xi \partial t_{1}}=\frac{\partial^{2} \varphi}{\partial t_{1} \partial \xi}=(\alpha-\beta p)\left\{\gamma p+\left(C_{d}-\eta C_{d}\right) \theta \omega^{\prime}(\xi)-C_{h}-C_{i}\left(\gamma+\theta \omega^{\prime}(\xi)\right)\right\} t_{1}$

The Hessian matrix for $\varphi\left(T, t_{1}, \xi\right)$ is $H=\left[\begin{array}{ccc}\frac{\partial^{2} \varphi}{\partial T^{2}} & \frac{\partial^{2} \varphi}{\partial t_{1} \partial T} & \frac{\partial^{2} \varphi}{\partial \xi \partial T} \\ \frac{\partial^{2} \varphi}{\partial T \partial t_{1}} & \frac{\partial^{2} \varphi}{\partial t_{1}^{2}} & \frac{\partial^{2} \varphi}{\partial \xi \partial t_{1}} \\ \frac{\partial^{2} \varphi}{\partial T \partial \xi} & \frac{\partial^{2} \varphi}{\partial t_{1} \partial \xi} & \frac{\partial^{2} \varphi}{\partial \xi^{2}}\end{array}\right]$

The first leading principal minor is $\left|H_{11}\right|=\frac{\partial^{2} \varphi}{\partial T^{2}}=-(\alpha-\beta p) \delta \frac{\left(p-C_{i}+\frac{C_{S}}{\delta}+C_{l}\right)}{\left\{1+\delta\left(T-t_{1}\right)\right\}^{2}}$ 
Since $(\alpha-\beta p)>0, \omega^{\prime \prime}(\xi)>0$ and $p-C_{i}>0$, so $\left|H_{11}\right|<0$

The second leading principal minor is

$$
\left|H_{22}\right|=\frac{\partial^{2} \varphi}{\partial t_{1}^{2}} \frac{\partial^{2} \varphi}{\partial T^{2}}-\frac{\partial^{2} \varphi}{\partial T \partial t_{1}} \cdot \frac{\partial^{2} \varphi}{\partial t_{1} \partial T}
$$

and, the third leading principal minor is $\left|H_{33}\right|=|H|=\left|\begin{array}{ccc}\frac{\partial^{2} \varphi}{\partial T^{2}} & \frac{\partial^{2} \varphi}{\partial t_{1} \partial T} & \frac{\partial^{2} \varphi}{\partial \xi \partial T} \\ \frac{\partial^{2} \varphi}{\partial T \partial t_{1}} & \frac{\partial^{2} \varphi}{\partial t_{1}^{2}} & \frac{\partial^{2} \varphi}{\partial \xi \partial t_{1}} \\ \frac{\partial^{2} \varphi}{\partial T \partial \xi} & \frac{\partial^{2} \varphi}{\partial t_{1} \partial \xi} & \frac{\partial^{2} \varphi}{\partial \xi^{2}}\end{array}\right|$

As $\left|H_{11}\right|<0$, so, if $\left|H_{22}\right|>0$ and $\left|H_{33}\right|<0$ then the Hessian matrix is negative definite. Therefore $\varphi\left(T, t_{1}, \xi\right)$ is negative, differentiable and strictly concave.

Moreover $\psi(\xi, T)=T$ is positive, differentiable, and convex function, so the average profit function per unit time $A P\left(T, t_{1}, \xi\right)$ is pseudo-concave function in $T, t_{1}, \xi$. Therefore $A P\left(T, t_{1}, \xi\right)$ attains the global maximum value at the point $\left(\xi^{*}, T^{*}, t_{1}^{*}\right)$ whenever $\left|H_{22}\right|>0$ and $\left|H_{33}\right|<0$.

\section{SOLUTION PROCEDURE AND ALGORITHM}

In this portion, solution process as well as algorithm have been drafted out to find optimal solution in price concession environment.

\section{IV.I Solution process.}

Retailer is getting offers from supplier unit purchasing cost $C_{i}$ $\left(C_{1}>C_{2}>\cdots>C_{n}\right)$ if the ordering quantity $(Q)$ lies in between $Q_{i}$ and $Q_{i+1}, i=1,2,3, \ldots, n\left(Q_{1}<Q_{2}<\cdots<Q_{n}<\right.$ $\left.Q_{n+1}=\infty\right)$. Since supplier offers less purchasing cost if retailer buys bigger amount of item that mentioned in assumption (viii) of section 2, so at first retailer will be interested to take the opportunity of lowest purchasing cost by purchasing more items in order to get his/her maximum profit. So, he/she will find,$t_{1}$ and $\xi$ from the equations (19), (20) and (21) by inserting all parameter values together with lowest purchasing $\operatorname{cost} C_{n}$, and then ordering quantity $Q$ from the equation (7). If $Q$ lies in between $Q_{n}, Q_{n+1}$, the solution $\left(T^{*}, t_{1}^{*}, \xi^{*}\right)$ is optimal and maximum profit will be $A P\left(T^{*}, t_{1}^{*}, \xi^{*}\right)$ (from equation (18)).

If $Q$ does not belong in between $Q_{n}, Q_{n+1}$, then the retailer will try to take benefit of minimum purchasing cost by taking ordering quantity $Q=Q_{n}$. So, setting $Q=Q_{n}$, in equation (8) he/she will get $T$, then the average profit function $A P$ will be a function of two variables $t_{1}$ and $\xi$ by substituting $T$ in equation (18). Consequently, there will be two decision variables $t_{1}$ and $\xi$ under which the profit function $A P\left(t_{1}, \xi\right)$ has to be maximized.

To obtain optimal $t_{1}$ and $\xi$, the necessary conditions are $\frac{\partial(A P)}{\partial t_{1}}=0$ and $\frac{\partial(A P)}{\partial \xi}=0$.

Calculating $\frac{\partial(A P)}{\partial t_{1}}$ and $\frac{\partial(A P)}{\partial \xi}$ by the help of equation (8)

$\frac{\partial T}{\partial t_{1}}=1-e^{(\gamma+\theta \omega(\xi)) t_{1}}\left[1+\delta\left(T-t_{1)}\right]\right.$

And $\frac{\partial T}{\partial \xi}=\frac{\theta \omega^{\prime}(\xi)}{(\gamma+\theta \omega(\xi))^{2}}\left[e^{(\gamma+\theta \omega(\xi)) t_{1}}\{1-(\gamma+\theta \omega(\xi))\}-\right.$

1] $\left[1+\delta\left(T-t_{1}\right)\right]$

and setting equal to zero, the necessary conditions are

$$
\left[\begin{array}{c}
(\alpha-\beta p)\left(p-C_{i}+\frac{C_{s}}{\delta}+C_{l}\right) T+(\alpha-\beta p)\left\{\gamma p+\left(C_{d}-\eta C_{d}\right) \theta \omega(\xi)-C_{h}-C_{i}(\gamma+\theta \omega)\right\} T t_{1} \\
+(\alpha-\beta p)\left(p-C_{i}+\frac{C_{s}}{\delta}+C_{l}\right) \frac{T}{1+\delta\left(T-t_{1}\right)}-\left[K+(\alpha-\beta p)\left(p-C_{i}+\frac{C_{s}}{\delta}+C_{l}\right) t_{1}+\right. \\
\frac{(\alpha-\beta p)}{2}\left\{\gamma p+\left(C_{d}-\eta C_{d}\right) \theta \omega(\xi)-C_{h}-C_{i}(\gamma+\theta \omega)\right\} t_{1}^{2}-(\alpha-\beta p)\left(p-C_{i}+\frac{C_{s}}{\delta}+C_{l}\right) \frac{T}{1+\delta\left(T-t_{1)}\right.} \\
\left.+\frac{(\alpha-\beta p)}{\delta}\left(p-C_{i}+\frac{C_{s}}{\delta}+C_{l}\right) \log \left\{1+\delta\left(T-t_{1}\right)\right\}\right] \times\left[1-e^{(\gamma+\theta \omega(\xi)) t_{1}}\left[1+\delta\left(T-t_{1)}\right]\right]\right.
\end{array}\right]=0
$$

and

$$
\left[\begin{array}{c}
\frac{(\alpha-\beta p)}{2}\left\{\left(C_{d}-\eta C_{d}\right) \theta \omega^{\prime}(\xi)-C_{h}-C_{i}\left(\gamma+\theta \omega^{\prime}(\xi)\right\} T t_{1}^{2}-T^{2}+(\alpha-\beta p)\left(p-C_{i}+\frac{C_{s}}{\delta}+C_{l}\right) \frac{T}{1+\delta\left(T-t_{1)}\right.}\right. \\
-\left[K+(\alpha-\beta p)\left(p-C_{i}+\frac{C_{s}}{\delta}+C_{l}\right) t_{1}+\frac{(\alpha-\beta p)}{2}\left\{\gamma p+\left(C_{d}-\eta C_{d}\right) \theta \omega(\xi)-C_{h}-C_{i}(\gamma+\theta \omega)\right\} t_{1}^{2}\right. \\
\left.-(\alpha-\beta p)\left(p-C_{i}+\frac{C_{s}}{\delta}+C_{l}\right) \frac{T}{1+\delta\left(T-t_{1}\right)}+\frac{(\alpha-\beta p)}{\delta}\left(p-C_{i}+\frac{C_{s}}{\delta}+C_{l}\right) \log \left\{1+\delta\left(T-t_{1}\right)\right\}\right] \times \\
\frac{\theta \omega^{\prime}(\xi)}{(\gamma+\theta \omega(\xi))^{2}}\left[e^{\left.(\gamma+\theta \omega(\xi)) t_{1}\{1-(\gamma+\theta \omega(\xi))\}-1\right]\left[1+\delta\left(T-t_{1)}\right]\right.}\right.
\end{array}\right]=0
$$


By means of equations (35), (36) and (8) retailer can catch on $T, t_{1}, \xi$ and $A P\left(T, t_{1}, \xi\right)$. If the profit is larger than the profit of the retailer by picking the next unit purchasing $\operatorname{cost} C_{n-1}$, then this $T, t_{1}, \xi$ will be optimal solution $\left(T^{*}, t_{1}^{*}, \xi^{*}\right)$, else repeat this procedure for the purchasing $\operatorname{cost} C_{n-1}$ and so on.

\section{IV.II Algorithm}

Here we present the algorithm for the model.

Step1. Set $\operatorname{Max} A T P\left(T, t_{1}, \xi\right)=-\infty$ and $i=n$.

Step 2. Insert all parameter values together with purchasing cost $C_{i}$ in equations (19), (20) and (21) to get $T, t_{1}$ and $\xi$. Evaluate $Q$ by substituting $T, t_{1}$ and $\xi$ in equation (7).

a) If $Q \in\left[Q_{i}, Q_{i+1}\right)$ then calculate $A P\left(T, t_{1}, \xi\right)$ (from equation(18)) and if $A P\left(T, t_{1}, \xi\right)>\operatorname{Max} A P\left(T, t_{1}, \xi\right)$, set $\operatorname{Max} A P\left(T, t_{1}, \xi\right)=A P\left(T, t_{1}, \xi\right)$. Go to step 5.

b) If $Q \notin\left[Q_{i}, Q_{i+1}\right)$ then go to step 3 .

Step 3. Set $Q=Q_{i}$ in equation (8). Put all values of parameters as well as $C_{i}$ and $T$ from equation (8) in equations (35) and (36) and solve for $t_{1}, \xi$. Calculate corresponding $T$ from equation (8) and lastly compute $A P\left(T, t_{1}, \xi\right)$ (from equation 18) with the support of these $T$ and $\xi$. If $A P\left(T, t_{1}, \xi\right)>\operatorname{Max} A P\left(T, t_{1}, \xi\right)$, set $\operatorname{Max} A P\left(T, t_{1}, \xi\right)=A P\left(T, t_{1}, \xi\right)$. Go to step 4.

Step 4. If $i \geq 2$, go to step 2 with $i=i-1$, else go to step 5 .

Step 5. Optimal profit is $\operatorname{Max} A P\left(T, t_{1}, \xi\right)$ with the corresponding $T, t_{1}$ and $\xi$.

\section{NUMERICAL ILLUSTRATIONS}

To exemplify different cases of the established model, three numerical samples are taken with their appropriate values.

Example 1. Let us take the following parameters in suitable units as follows:

Price breaks:

\begin{tabular}{|c|c|c|c|}
\hline Ordering quantity & $0=Q_{1} \leq Q<Q_{2}=300$ & $300=Q_{2} \leq Q<Q_{3}=550$ & $550=Q_{3} \leq Q<=\infty$ \\
\hline Unit purchase cost & $C_{1}=\$ 12.25$ & $C_{2}=\$ 11.5$ & $C_{3}=\$ 10.75$ \\
\hline
\end{tabular}

$\&$ all other parameters are $K=\$ 1500, \alpha=80, \beta=1.5$, $\gamma=0.03, C_{h}=\$ 0.80$ per unit item per month, $p=\$ 20$ per unit item, $C_{d}=\$ 1.0$ per unit item, $\theta=0.25$, $\eta=0.08 \delta=0.02, C_{s}=\$ 5$ per unit item, $C_{l}=\$ 11$ per unit item and $\omega(\xi)=e^{-v \xi}$, where $v=2$. The values of the parameters are considered here are realistic, though these values are not taken from any case study of an existing inventory problem. The computational effort has been completed by MATHEMATICA 12.
Maximum profit per month for this example can be evaluated by the help of algorithm discussed in section 4.2 as follows:

Step 1: Set $\operatorname{Max} A P(T, \xi)=-\infty$ and $i=3$

Step 2: Inserting all values of parameters including $C_{3}=\$ 10.75$ in equations (19), (20) and (21), we obtain $T=10.3042 \quad t_{1}=9.24743$ and $\xi=3.59073$ and the corresponding ordering quantity $Q=585.652$. Inserting these values of $T, t_{1}$ and $\xi$ in equation (18), we get $A P\left(T, t_{1}, \xi\right)=$ 179.23. Since $Q=585.652 \in[550, \infty)$ and $A P\left(T, t_{1}, \xi\right)=$ $179.23>\operatorname{Max} A P\left(T, t_{1}, \xi\right)=-\infty, \quad$ so we set $\operatorname{Max} A P\left(T, t_{1}, \xi\right)=179.23$ and go to step 5 .

Step 3. Optimal solution is $T^{*}=10.3042$ month, $t_{1}^{*}=$ 9.24743 month and $\xi^{*}=\$ 3.59073, Q^{*}=585.652$ unit and $\operatorname{Max} A P\left(T, t_{1}, \xi\right)=\$ 179.23$.

The concavity of the profit function against $\left(T, t_{1}\right),(T$, $\xi)$ and $\left(t_{1}, \xi\right)$ are shown separately in Fig 2, Fig 3 and Fig 4 respectively.

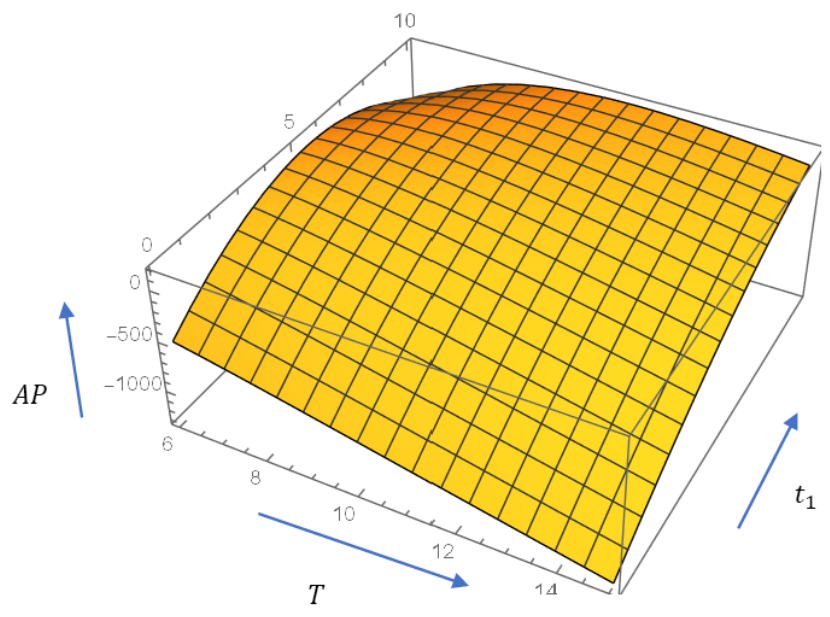

Fig 2. Profit per unit time vs $T$ and $t_{1}$ of example 1

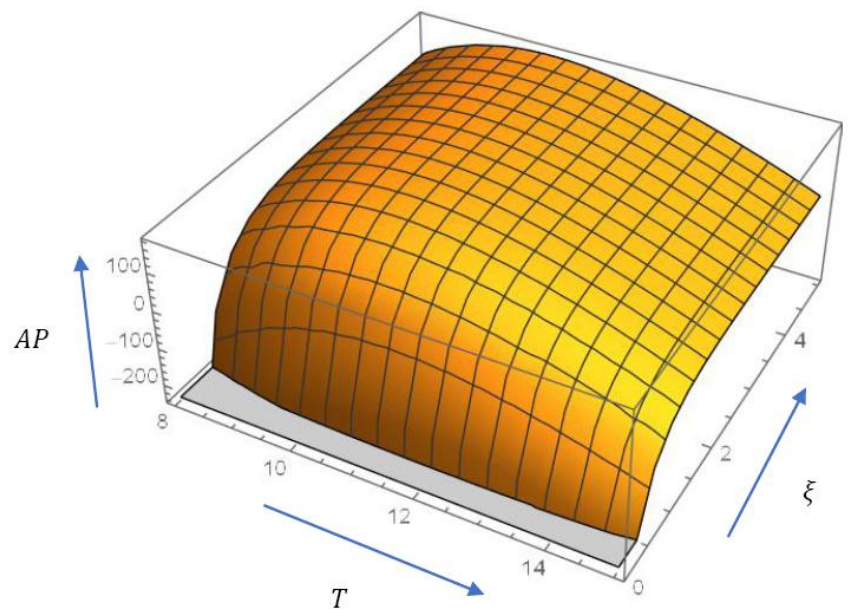

Fig 3. Profit per unit time vs $T$ and $\xi$ of example 1 


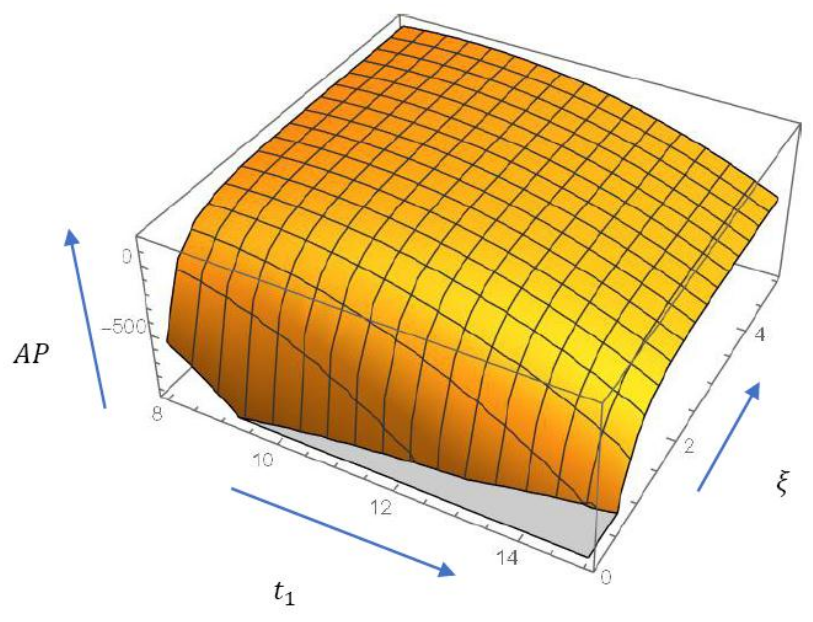

Fig 4. Profit per unit time vs $t_{1}$ and $\xi$ of example 1

Example 2. All data are same as of example 1 except $\alpha=65$ Here also maximum profit per month can be computed by the help of algorithm discussed in section 4.2 as follows:

Step 1: Set $\operatorname{Max} A P(T, \xi)=-\infty$ and $i=3$

Step 2: Inserting all values of parameters including $C_{3}=$ $\$ 10.75$ in equations (19), (20) and (21), we obtain $T=$ $12.1341 t_{1}=10.8555$ and $\xi=3.51285$ and the corresponding ordering quantity $Q=493.862$. Inserting these values of $T, t_{1}$ and $\xi$ in equation (18), we get $A P\left(T, t_{1}, \xi\right)=$ 84.3091. Since $Q=493.862 \notin[550, \infty)$, it is not feasible. Hence go to step 3.

Step3. Set $Q=550$ and put all values of parameters including $C_{3}=\$ 10.75$ in equations (35) and (36). Solving equations (35) and (36) by the help of equation (8), we get $T=$ $13.28182 t_{1}=11.95776$ and $\xi=3.57937$.

Finally, using these $T, t_{1}$ and $\xi$ in equation (17) we have found $A P\left(T, t_{1}, \xi\right)=52.2478$. Since $\quad A T P\left(T, t_{1}, \xi\right)=$ 52.2478> $\operatorname{Max} A P(T, \xi)$, set $\operatorname{Max} A P(T, \xi)=52.2478$. Go to step 4.

Step 4. Set $i=2$. Using all parameters including $C_{2}=\$ 11.50$ in equations (19), (20) and (21), we obtain $T=11.9367 t_{1}=$ 10.6306 and $\xi=3.53129$ and the corresponding ordering quantity $Q=483.897$ Inserting these values of $T, t_{1}$ and $\xi$ in equation (18), we get $A P\left(T, t_{1}, \xi\right)=53.8451$. Since $Q=$ $483.897 \in[350,550) \quad$ and $A P\left(T, t_{1}, \xi\right)=53.8451>$ $\operatorname{Max} A P(T, \xi)=52.2478, \quad$ so we set $\operatorname{Max} A P(T, \xi)=$ 53.8451 and optimal solution is corresponding $\quad T=$ $11.9367, t_{1}=10.6306, \xi=3.53129$ and $Q=483.897$. Go to step 5 .

Step 5. Optimal solution is $T^{*}=11.9367$ month, $t_{1}^{*}=$
10.6306 month and $\xi^{*}=\$ 3.53129, Q^{*}=483.897$ unit and $\operatorname{Max} A P\left(T, t_{1}, \xi\right)=\$ 53.8451$

Example 3. All data are same as of example 1 except $\omega(\xi)=$ $\frac{1}{1+v \xi}$, where $v=2$

Here also maximum profit per month can be computed by the help of algorithm discussed in section 4.2 as follows:

Step 1: Set $\operatorname{Max} A P(T, \xi)=-\infty$ and $i=3$

Step 2: Inserting all values of parameters including $C_{3}=$ $\$ 10.75$ in equations (19), (20) and (21), we obtain $T=8.9394$ $t_{1}=7.7289$ and $\xi=7.001$ and the corresponding ordering quantity $Q=525.134$. Inserting these values of $T, t_{1}$ and $\xi$ in equation (18), we get $A P\left(T, t_{1}, \xi\right)=136.125$. Since $Q=$ $525.134 \notin[550, \infty)$, it is not feasible. Hence go to step 3 .

Step3. Set $Q=550$ and put all values of parameters including $C_{3}=\$ 10.75$ in equations (35) and (36). Solving equations (35) and (36) by the help of equation (8), we get $T=9.6754$ $t_{1}=8.7564$ and $=7.3542$.

Finally, using these $T, t_{1}$ and $\xi$ in equation (17) we have found $A P\left(T, t_{1}, \xi\right)=82.3498$. Since $\quad \operatorname{ATP}\left(T, t_{1}, \xi\right)=$ 82.3498> $\operatorname{Max} A P(T, \xi)$, set $\operatorname{Max} A P(T, \xi)=82.3498$. Go to step 4.

Step 4. Set $i=2$. Using all parameters including $C_{2}=\$ 11.50$ in equations (19), (20) and (21), we obtain $T=8.77674 t_{1}=$ 7.53782 and $\xi=7.00$ and the corresponding ordering quantity $Q=512.878$ Inserting these values of $T, t_{1}$ and $\xi$ in equation (18), we get $A P\left(T, t_{1}, \xi\right)=92.1836$. Since $Q=512.878 \in$ $[350,550)$ and $A P\left(T, t_{1}, \xi\right)=92.1836>\operatorname{Max} A P(T, \xi)=$ 82.3498, so we set $\operatorname{Max} A P(T, \xi)=$ 92.1836 and optimal solution is corresponding $\quad T=$ 8.77674, $t_{1}=7.53782, \xi=7.00$ and $Q=512.878$. Go to step 5 .

Step 5. Optimal solution is $T^{*}=8.77674$, month, $t_{1}^{*}=$ 7.537826 month and $\xi^{*}=\$ 7.00, Q^{*}=512.878$ unit and $\operatorname{MaxAP}\left(T, t_{1}, \xi\right)=\$ 53.8451$

\section{SENSITIVITY ANALYSIS}

To check the flexibility of the model, a study of the impact of changes in different parameters is made against optimal solutions $\left(T, t_{1}, \xi\right)$, optimal ordering quantities and average profit for the example1. Changing the value on one parameter by $-20 \%$ to $+20 \%$ at a time and fixing other remaining parameters, the analysis has been done. In the following Table 1 , the result of this sensitivity analysis is given . 
International Journal of Engineering Research and Technology. ISSN 0974-3154, Volume 13, Number 6 (2020), pp. 1420-1431

(C) International Research Publication House. https://dx.doi.org/10.37624/IJERT/13.6.2020.1420-1431

Table 1. Sensitivity Analysis

\begin{tabular}{|c|c|c|c|c|c|c|c|}
\hline Parameter & $\begin{array}{c}\text { Original } \\
\text { value }\end{array}$ & New value & $\boldsymbol{T}^{*}$ & $t_{1}^{*}$ & $\xi^{*}$ & $Q^{*}$ & $A P$ \\
\hline \multirow{4}{*}{$K$} & \multirow{4}{*}{1500} & 1800 & 11.2091 & 10.0447 & 3.64210 & 644.2 & 151.344 \\
\hline & & 1650 & 10.7685 & 9.65683 & 3.61754 & 615.541 & 164.994 \\
\hline & & 1350 & 9.81257 & 8.81317 & 3.56121 & 554.35 & 194.142 \\
\hline & & 1200 & 9.28882 & 8.34968 & 3.52834 & 550.00 & 211.847 \\
\hline \multirow{4}{*}{$\alpha$} & \multirow{4}{*}{80} & 96 & 9.06031 & 8.1482 & 3.65231 & 669.301 & 287.301 \\
\hline & & 88 & 9.62975 & 8.6441 & 3.62355 & 628.927 & 232.572 \\
\hline & & 72 & 11.1643 & 10.0045 & 3.55248 & 538.947 & 127.626 \\
\hline & & 64 & 12.2952 & 10.996 & 3.50658 & 487.095 & 78.291 \\
\hline \multirow{4}{*}{$\beta$} & \multirow{4}{*}{1.5} & 1.80 & 10.9286 & 9.79725 & 3.56265 & 550.864 & 140.339 \\
\hline & & 1.65 & 10.6022 & 9.51000 & 3.57712 & 568.537 & 159.651 \\
\hline & & 1.35 & 10.0307 & 9.00616 & 3.60359 & 602.262 & 199.051 \\
\hline & & 1.20 & 9.77843 & 8.78343 & 3.61577 & 618.404 & 219.094 \\
\hline \multirow{4}{*}{$\gamma$} & \multirow{4}{*}{0.03} & 0.036 & 10.6105 & 9.59537 & 3.62038 & 623.828 & 190.008 \\
\hline & & 0.033 & 10.4511 & 9.41476 & 3.60515 & 603.856 & 184.508 \\
\hline & & 0.027 & 10.1682 & 9.09183 & 3.57703 & 568.972 & 174.155 \\
\hline & & 0.024 & 10.0420 & 8.94666 & 3.56399 & 553.628 & 169.268 \\
\hline \multirow{4}{*}{$C_{h}$} & \multirow{4}{*}{0.80} & 0.96 & 9.26187 & 8.07573 & 3.51218 & 515.943 & 145.861 \\
\hline & & 0.88 & 9.73198 & 8.60776 & 3.54937 & 547.111 & 161.810 \\
\hline & & 0.72 & 11.0209 & 10.0384 & 3.63766 & 634.875 & 198.458 \\
\hline & & 0.64 & 11.9545 & 11.0549 & 3.69239 & 700.609 & 219.996 \\
\hline \multirow{4}{*}{$p$} & \multirow{4}{*}{20} & 24 & 12.1299 & 11.1352 & 3.63633 & 625.642 & 343.991 \\
\hline & & 22 & 11.1317 & 10.1036 & 3.61213 & 603.062 & 267.348 \\
\hline & & 18 & 9.60548 & 8.52359 & 3.5714 & 571.944 & 79.5647 \\
\hline & & 16 & 9.00664 & 7.9025 & 3.55365 & 560.98 & -31.6949 \\
\hline \multirow{4}{*}{$C_{d}$} & \multirow{4}{*}{1.0} & 1.2 & 10.3042 & 9.24739 & 3.58268 & 585.659 & 179.238 \\
\hline & & 1.1 & 10.3042 & 9.24741 & 3.58673 & 585.656 & 179.234 \\
\hline & & 0.9 & 10.3042 & 9.24745 & 3.59471 & 585.649 & 179.226 \\
\hline & & 0.8 & 10.3042 & 9.24747 & 3.59865 & 585.645 & 179.222 \\
\hline \multirow{2}{*}{$\eta$} & \multirow{2}{*}{0.08} & 0.096 & 10.3042 & 9.24743 & 3.59142 & 585.652 & 179.229 \\
\hline & & 0.088 & 10.3042 & 9.24743 & 3.59108 & 585.652 & 179.229 \\
\hline
\end{tabular}


International Journal of Engineering Research and Technology. ISSN 0974-3154, Volume 13, Number 6 (2020), pp. 1420-1431

(C) International Research Publication House. https://dx.doi.org/10.37624/IJERT/13.6.2020.1420-1431

\begin{tabular}{|c|c|c|c|c|c|c|c|}
\hline Parameter & $\begin{array}{c}\text { Original } \\
\text { value }\end{array}$ & New value & $\boldsymbol{T}^{*}$ & $t_{1}^{*}$ & $\xi^{*}$ & $\boldsymbol{Q}^{*}$ & $A P$ \\
\hline & & 0.072 & 10.3042 & 9.24743 & 3.59038 & 585.653 & 179.230 \\
\hline & & 0.064 & 10.3042 & 9.24743 & 3.59004 & 585.653 & 179.230 \\
\hline \multirow{4}{*}{$\theta$} & \multirow{4}{*}{0.025} & 0.3 & 10.3042 & 9.24743 & 3.68189 & 585.652 & 179.138 \\
\hline & & 0.275 & 10.3042 & 9.24743 & 3.63838 & 585.652 & 179.182 \\
\hline & & 0.225 & 10.3042 & 9.24743 & 3.53805 & 585.652 & 179.282 \\
\hline & & 0.20 & 10.3042 & 9.24743 & 3.47916 & 585.647 & 179.341 \\
\hline \multirow{4}{*}{$v$} & \multirow{4}{*}{2} & 2.4 & 10.3545 & 9.28765 & 3.35298 & 585.650 & 179.454 \\
\hline & & 2.2 & 10.3275 & 9.26321 & 3.45376 & 585.657 & 179.328 \\
\hline & & 1.8 & 10.2143 & 9.21421 & 3.77543 & 585.660 & 179.156 \\
\hline & & 1.6 & 10.1987 & 9.18763 & 3.89765 & 585.652 & 179.103 \\
\hline \multirow{4}{*}{$\delta$} & \multirow{4}{*}{0.02} & 0.024 & 10.2984 & 9.25250 & 3.59163 & 585.348 & 179.055 \\
\hline & & 0.022 & 10.3013 & 9.24998 & 3.59118 & 585.500 & 179.142 \\
\hline & & 0.018 & 10.3072 & 9.24485 & 3.59027 & 585.810 & 179.318 \\
\hline & & 0.016 & 10.3102 & 9.24224 & 3.58981 & 585.969 & 179.408 \\
\hline \multirow{4}{*}{$C_{s}$} & \multirow{4}{*}{5} & 6 & 10.2098 & 9.31365 & 3.60335 & 582.144 & 176.951 \\
\hline & & 5.5 & 10.2530 & 9.28319 & 3.59775 & 583.749 & 178.000 \\
\hline & & 4.5 & 10.3657 & 9.24049 & 3.58257 & 587.939 & 180.692 \\
\hline & & 4 & 10.4409 & 9.15334 & 3.57266 & 590.732 & 182.459 \\
\hline \multirow{4}{*}{$C_{l}$} & \multirow{4}{*}{11} & 13.20 & 10.2993 & 9.25082 & 3.59138 & 585.47 & 179.113 \\
\hline & & 12.10 & 10.3017 & 9.24913 & 3.59106 & 585.559 & 179.171 \\
\hline & & 9.90 & 10.3067 & 9.24571 & 3.5904 & 585.745 & 179.289 \\
\hline & & 8.80 & 10.3091 & 9.24348 & 3.59007 & 585.834 & 179.348 \\
\hline \multirow{4}{*}{$C_{i}$} & \multirow{4}{*}{$\begin{array}{l}12.25 \\
11.50 \\
10.75\end{array}$} & $\begin{array}{c}14.7, \quad 13.8 \\
12.9\end{array}$ & 9.83926 & 8.71905 & 3.64119 & 554.065 & 57.6109 \\
\hline & & $\begin{array}{c}13.475,12.65 \\
11.825\end{array}$ & 10.0617 & 8.97278 & 3.61755 & 559.123 & 118.279 \\
\hline & & $\begin{array}{c}11.025,10.35 \\
9.675\end{array}$ & 10.57 & 9.54621 & 3.55999 & 603.91 & 240.486 \\
\hline & & $\begin{array}{c}9.8,9.235 \\
8.6\end{array}$ & 10.8629 & 9.87303 & 3.52433 & 624.201 & 302.079 \\
\hline
\end{tabular}

From Table 1, the following observations and economic interpretations can be made that can be suggested to the retailer.

(i) The replenishment cost $(K)$ has positive significant impact on the cyclic period $(T)$, positive inventory period $\left(t_{1}\right)$ and the ordering quantity $(Q)$, while it has significant negative impact on the profit $(A P)$. Also preservation cost has moderate positive impact on the 
replenishment cost $(K)$. Consequently, if the ordering cost is high the retailer will take opportunity of price discount by buying big amount of product at a time and invest more amount to preserve deteriorating items.

(ii) The parameter $\alpha$ has significant role on determining the cyclic period $(T)$, positive inventory period $\left(t_{1}\right)$, preservation cost $(\xi)$ and the ordering quantity $(Q)$. When $\alpha$ is high, the profit $(A P)$, ordering quantity $(Q)$, preservation cost $(\xi)$ are high, while cyclic period $(T)$, positive inventory period $\left(t_{1}\right)$ are low. Therefore, if the demand is high, retailer will earn more profit as expected, take price discount benefit as higher number of products has to be replenished at the beginning of the cycle and invest to preserve items. Cycle time and positive inventory time will be obviously of shorter length as demand is high.

(iii) The parameter $\beta$ has also significant role on $T, t_{1}, Q, \xi$ and profit. The table shows that, if $\beta$ is high, the profit $(A P)$, ordering quantity $(Q)$, preservation cost $(\xi)$ are low, while cyclic period $(T)$, positive inventory period $\left(t_{1}\right)$ are high. Therefore, if the demand is highly price sensitive, retailer will earn less profit as expected, lower number of products has to be replenished and so, investment cost to preserve items will be less and he will not be interested to take benefit of price discount. Cycle time and positive inventory time will be obviously of longer as demand is low.

(iv) Table 1 indicates, the stock dependent demand rate parameter $\gamma$ has positive impact on all decision variables $\left(T, t_{1}, \xi\right)$, profit and ordering quantity. Which is realistic because when the demand is very much stock dependent, obviously retailer will order more products at the starting point of the cycle to display on the shelf and as a result more investment is required to preserve them and price discount facility will be available. Consequently, positive inventory period as well as cycle time will be longer.

(v) From the table, we observe that inventory holding cost $\left(C_{h}\right)$ has strong negative impact on the result of all. Which is realistic because when a retailer needs to invest more money to hold items, then he/she will not be interested to preserve items and so a smaller number of items will be replenished and fails to take benefit of price discount. Consequently, positive inventory period as well as cycle time will be shorter and hence less profit will be accumulated.

(vi) As expected, selling price of the product $(p)$ has huge positive impact on the result. If the selling price is high then retailer will take discount facility by replenishing bigger amount, invest to preserve items and as a result positive inventory period, cycle time will be longer and hence more profit will be accumulated.

(vii) It is noticed from this analysis that, variation in deterioration rate do not have a strong impact on the result except preservation cost. Preservation cost is increased due to increasing value of deterioration. If the product deteriorates at a high rate, then the retailer is suggested to reduce the deterioration rate by increasing investment cost for preservation technology.

(viii) The parameter $v$ has negative effect on preservation cost. However no remarkable effect is seen on others.

(ix) Purchase cost $\left(C_{i}\right)$ has a huge emphasis in determination of $T, t_{1}, Q, \xi$ and profit. If purchase cost $\left(C_{i}\right)$ increase, then preservation cost increases and all others i.e., $T, t_{1}, Q$, profit are decreased. So, retailer wants to preserve items as the items are costly.

(x) From the table, we readily observed that there is no noticeable effect on profit and others due to the small variation of other parameters $C_{d}, \eta, \delta, C_{s}$ and $C_{l}$.

\section{CONCLUSIONS}

In this article, we discussed the effect of price discount and preservation technology on an EOQ problem according to stock and price depending on demand with partial backlogging. Expression of the average profit function is derived by formulating the problem mathematically. The solution procedure and algorithm are introduced to determine optimal cycle time and optimal preservation cost. With the help of MATHEMATICA 12 software three different numerical example are demonstrated for illustration purpose. The concave nature of the profit function is justified by drawing graphs in three dimensions. To check the changes in the decision variables for changes in different parameters, a sensitivity analysis is also carried out.

This model can also be enriched by incorporating time dependent holding cost, inflation and trade credit financing. Finally, someone can extend this deterministic model to probabilistic model.

\section{REFERENCES}

[1] Haris, F. How many parts to make at once, Factory. The magazine of Management. 10(2), (1913), 135-136

[2] Levin, R.I., McLaughlin, C.P., Lamone, P.P., Kohas, J.F. Complementary policy for managing operating systems. New York, McGraw-Hill, (1972), 373.

[3] Padmanabhan, G., and Vrat, P. Analysis of multi-item inventory systems under resource constraints: A nonlinear goal programming approach. Engineering Costs and Production Economics. 20, (1990), 121-127.

[4] Giri, B.C., Pal, S., Goswami, A., Chaudhri, K.S. An inventory model for deteriorating items with stockdependent demand rate. European Journal of Operations Research. 95(3), (1996), 604-610.

[5] Kotler, P. Marketing Decision Making: A model building approach. New York: Holt Rinehart \& Winston (1971).

[6] Urban, T.L., Baker, R.C. Optimal ordering and pricing policies in a single-period environment with multivariate demand and markdowns. European Journal 
of Operational Research Society. 103, (1997),573- 583

[7] Whitin, T.M. The theory of inventory management, 2nd edition. Princeton, N.J: Princeton University Press (1957).

[8] Ghare, P.M., Schrader, G. F. A model for an exponentially decaying inventory. Journal of Industrial Engineering. 14, (1963),238-243.

[9] Hsu, P.H., Wee, H.M., Teng, H.M. Preservation technology investment for deteriorating inventory. International Journal of Production Economics. 124(2), (2010), 388-394

[10] Lee, Y. P., Dye, C. Y., An inventory model for deteriorating items under stock dependent demand rate and controllable deterioration rate. Computer \& Industrial Engineering. 63(2), (2012), 474-482.

[11] Mishra, V.K. An inventory model for instantaneous deteriorating items with controllable deterioration rate for time dependent demand \& holding cost. Journal of Industrial Engineering \& Management. 6(2), (2013), 495-506.

[12] Mashud A. H.M., Hasan, M.R., Wee, H. M., Daryanto Y. Non-instantaneous deteriorating inventory model under the joined effect of trade credit, preservation technology and advertisement policy: Kibernates in Press doi 10.118/K-2019-0357 (2019)

[13] Goh, M \& Sharafali, M. Price-dependent inventory models with discount offers at random times. Production \& Operations Management. 11(2), (2002), 139-156.

[14] Taleizadah A.A., Pentico, D. W. An economic order quantity model with partial backlogging and all unit discount. International Journal of Production Economics. 155, (2014), 172-184.

[15] Taleizadeh, A.A., Stojkovska, I., Pentico, D.W. An economic order quantity model with partial backlogging and incremental discount. Computer \& Industrial Engineering. 82, (2015), 21-32.

[16] Shah, N. H., Naik, K.M. Inventory policies for pricesensitive stock dependent demand and quantity discounts. International Journal of Mathematics, Engineering and Management Sciences. 3, (2018), 245257.

[17] Noori-darayan, M., Taleizadeh, A.A., Govidan, K. Joint replenishment and pricing decisions with different freight modes considerations for a supply chain under a composite incentive contact. Journal of the Operational Research Society 69(6), (2018),876-894.

[18] Pervin, M., Roy, S.K., Weber G.H. Deteriorating inventory with preservation technology under-pricestock sensitive demand. American Institute of mathematical sciences. doi 10.3934/ijimo.2019019(2019).

[19] Guiping, L., Xiuli, H., Jing, Z., Hao, W. Pricing, replenishing \& preservation technology investment for non-instantaneous deteriorating items. Omega. 84®, (2019), 114-126.

[20] Alfares H.K., Ghaithan, A.M. Inventory and pricingdependent demand, time varying holding cost and quantity discount. Computer \& Industrial Engineering 94, (2016), 170-177

[21] Shaikh A.A., Khan M.A.A., Panda G.C., Konstantaras, I. Price discount facility in an EOQ model for deteriorating items with stock-dependent demand \& partial backlogging. International Journal of Operational Research. 26, (2019), 1365-1395.

[22] Combini, A., Martain L. Generalized Convexity \& Optimization: Theory and application. Springer- Verlag, Berlin(2009).

[23] Dye C Y. The effect of preservation technology investment on a non-instantaneous deteriorating inventory model. Omega 41(5), (2013), 872-880. 